his experiments on inclastic scattering with energy transfors of several $\mathrm{BeV}$. Unlike the elastic electron proton scattering, and the seattering leading to definite excited nucleon states, this "decp" inelastic scattering varies only slowly with momentum transfer. This therefore describes a structure at distances smaller than the proton sizo. Various theoretical attempts to describe this have been made; it scems that the electrons are scattered by a small part of the nuclous; the constituent is commutally callod a "parton".

The voctor meson production by electron-positron colliding beams has been extended in the last year. There now exists good measurements on the $p$ meson, detected by its usual $\pi^{+} \pi$ - decay; the $\omega$, delected both by its $\pi^{+} \pi^{-} \pi^{0}$ decay and a rare $\pi^{+} \pi^{-*}$ decay; and the $\varphi$ detected now by $\mathrm{K}^{+} \mathrm{K}^{-}, \mathrm{K}_{1}^{0} \mathrm{~K}_{2}^{0}$ and $\pi^{+} \pi^{-} \pi^{0}$ decays. All of these give a consistent picture. The coupling of each vector meson to the photon field is measured and seems to be, within errors, equal to that assumed in the model of Vector Meson Dominance (VMD), first proposed by Sakurai, in which the $\omega, p$ and $\varphi$ mesons are assumed to dominate the electromagnetic interactions. Dr Aleock presented one of the first experiments from the Daresbury Laboratory showing the interference between the leptonic decays of the $\omega$ and $p$ mesons. Much work has bcen done in the past year to establish, both theoretically and experimentally, the limits of validity of the VMD model. Deviations aro obscrved but VMD remains a very useful concept.

Expcriment and theory now agree that quantum electrodynamics describes puroly electromagnetic processes down to distances of the order of $10^{-15} \mathrm{~cm}$. At the conference, Dr Brodsky described the recent advances; experiments of bremsstrahlung and pair production by electron and muons at high momentum transfers agree well with calculation; the difference of the $g$ factor of the free muon from the Dirac value of 2 , measured at CERN by Dr Farley and collaborators, now agrees with theory. $\Lambda$ recont calculation of a correction term by Dr Kinoshita and collaborators-a term involving scattering of light by light-brings a new and welcome agreement. Since the conference, and therefore not in the proceedings, a recalculation of the 4th order corrections to the Lamb shift has been made, which removes another discrepancy. Everybody in the fiold will nced a copy of these proceed. ings near them (till the next conference) and others would do well to read it.

RichaRd WILSON

\section{NEW MATHS}

A Comprehensive Textbook of Classical Mathematics A Contemporary Interpretation. By H. B. Griffiths and P. J. Hilton. Pp. xxix +637 . (Van Nostrand Reinhold: London and New York, February 1970.) 120s.

This is a splendid introduction to the "new maths". "Comprehensive" is a bit grand-the material is more or less at first year undergraduate level, and the book grew from a course of lectures to school teachers, who wanted to be up-dated with the new language of sets and functions. "It has not been our primary intention to offer readers their first contact with the concopts and ideas we treat," so that the course is heavily didactic. The authors are not against discovery methods; the creative aspect is important, but here they are jolly well telling us how one acquires strength through unification. They have ducked the important question as to how far the new notations and treatments might be used for the actual introduction of topics, at school level, but perhaps they can reasonably argue that this is not their problem.

The book is in eight parts. Tho first two sot up tho language of set theory, and the last introduces categories and ends with a chapter on mathematical logic. In between, first, there is arithmetic, generalizing from the integers to rings with a norm. Ncxt, there is three-dimen sional geometry, introduced with vectors and leading on to lincar algebra. The final chapter of this part is devoted to projective geometry. Durcll would not have recognized the language, but the authors justify their "commercial" at the beginning for the excellence of projective geometry in showing what genuine mathematics is. (Incidentally, the definition of cross-ratio "where $|\mathrm{PQ}|$ here is - $|\mathrm{QP}|$ ", reads a bit strangely-this seems to ariso from an ambiguity on p. 188.) Part five is devoted to algebra (groups, vector spaces, Boolean algebra, polynomials); and part six to number systems and topology (successive build-up to complex numbers and on to metric spaces).

The remaining part soven is concerned with calculus. The authors are at the same time humane teachers and puritanical mathematicians. The reader is edged forward with the spiral approach, but extrinsic motivation is minimal (tho logarithm, for example, is starkly defined as an integral). But then this is a course for the already converted. Is there any danger that the fascination of compact language will lead, not to unification and clarity, but to confusion? Certainly no schoolboy at the stagc of discovering that every differentiable function is continuous is going to thank his teacher for the theorem:

$\mathrm{D}(\mathrm{I})$ is a sub-algebra (over $\mathrm{R}$ ) of $\mathrm{C}(\mathrm{I})$, containing the constants and the identity function $I$.

Will even undergraduates appreciato this particular formulation?

But, by and large, the new look has been amply justified and agreeably presented. The abovo Michelin-type summary can give no roal impression of the scholarship and onjoyment clearly shared and imparted by the authors.

Geoffrey MatThews

\section{ELEMENTARY EQUATIONS}

\section{Elementary Differential Equations}

By R. L. E. Schwarzenberger. (Chapman and Hall Mathematics Series.) Pp. xii +98 . (Chapman and Hall: London, September 1969.) $20 \mathrm{~s}$.

This introductory textbook is based on a course of twenty lectures given at the University of Warwick to first year students. The motivation is clear and admirable: to base the course on geometric intuition and to be concrete rather than abstract. The former is in the spirit of a great deal of contemporary research in the field of differential equations and the concrote approach achieves two objects; first, it means that the course is more dircetly related to school mathematics, as experienced by most pupils, and second, it leads, in a natural manner, to various abstract notions such as continuous function, vector space and linear operator.

The exposition commences with an account of differentiable functions and with some examples of differential equations. A large number of diagrams illustrate the text, this being in the spirit of the geometric approach. Then the solution of first order equations is discussed thoroughly. Possibly the most interesting and important chapter is the one relating to autonomous systems. The frequent occurrence of such systems in physical contexts is pointed out and the geometric representation of the solutions is emphasized.

The book closes with a guide to further reading, particularly in the mathematical theory, and exercises are set at the end of each chapter. The student should find the book eminently readable, and it is reasonably priced It is refreshing, in view of the large number of texts in recent years on the subject of differential equations, tc find one that is different in emphasis from most of the others.
L. S. GODdARD 